\title{
REPARAÇÃO TRAQUEAL EM CÃES: TRANSPLANTE AUTÓGENO VS IMPLANTE HOMÓGENO CONSERVADO EM GLICERINA A 98\% DE CARTILAGEM DA PINA
}

\author{
TRACHEAL HEALING IN DOGS: AUTOGRAFT VERSUS HOMOGRAFT IMPLANT \\ PRESERVED IN 98\% GLYCERIN OF PINNA CARTILAGE
}

\author{
Emerson Antonio Contesini ${ }^{1}$ Mônica Seabra Salles ${ }^{2}$ João Antônio Tadeu Pigatto ${ }^{3}$ \\ Ney Luis Pippi ${ }^{4}$ Alceu Gaspar Raiser ${ }^{5}$
}

RESUMO

Diversas patologias acometem a traquéia dos animais domésticos de forma a comprometer o fluxo respiratório, inclusive a sobrevivência do animal. Obstrução por corpo estranho ou neoplasias, rupturas traumáticas, colapso e estenose são tidas como as principais. Reparações de defeitos induzidos experimentalmente em traquéias de 12 cães foram testadas comparativamente com o emprego de retalho de cartilagem da pina autógena em 6 cães (grupo I) e homógena conservada em glicerina a $98 \%$ nos demais (grupo II). Para tal, removeu-se um terço da circunferência de três anéis da traquéia cervical. Esses animais permaneceram durante um período de 30 e 45 dias sob observação e avaliação clínica em descanso e exercício físico moderado, sem apresentar quaisquer complicações decorrentes da cirurgia. Ao final desse período, o segmento traqueal operado foi submetido à avaliação macro e microscópica, sendo observado macroscopicamente, encobrimento completo do retalho por uma estrutura delgada e esbranquiçada em todos os animais. A análise microscópica da região transplantada do grupo I revelou que a mesma foi invadida por tecido de granulação, a lâmina própria apresentou moderada infiltração de neutrófilos e pouca fibrose com focos de hiperplasia epitelial. Nos animais do grupo II, foram observadas, na região do implante, além disso, discreta invasão de macrófagos e vasos neoformados, com inflamação moderada na lâmina própria, $e$ epitélio sem alteração. Essas observações sugerem que o implante de cartilagem da pina conservada em glicerina a 98\%, induz resposta menos agressiva pelo leito receptor, embora isso não seja evidenciado macroscopicamente.

Palavras-chave: cirurgia, sistema respiratório, cicatrização traqueal.

\section{SUMMARY}

Several patologies affect the trachea, compromising the respiratory stream and the animal survival. Foreign bodies, neoplasm, ruptures, collapse and stricture are the most common ones. The effects of canine pinna cartilage implantation were evaluated in 12 dogs. Six of them were submitted to an autograft (group I) and six to homograft preserved in $98 \%$ glycerin (group II), to repair tracheal defects. One third of three cervical tracheal rings were removed and substituted for auto or homograft. These animals remained under observation and clinical evaluation in rest and moderate physical exercise during a 45 days period, without presenting any decurrent complications. At the end of the observation period, the trachea was submitted to gross and microscopic examination. Full epithelization of the grafted area was observed by macroscopic evaluation in both groups. Microscopic analyses showed granulation tissue in the transplanted area of group I, the lamina propria with moderate infiltration of neutrophile and few fibrosis with focus of epithelial hyperplasia. Animals of group II presented granulation around the area of the implantation and discrete invasion of macrophages and neomorph vases in the lamina propria, with moderate inflammation. The epithelium showed no alteration. These data suggest that the pinna cartilage homograft implant induce less aggressive response by the receiving streambed, even so this was not observed grossly.

Key words: surgery, respiratory system, tracheal healing.

\section{INTRODUÇÃO}

A reparação de defeitos ou lesões traqueais tem sido de interesse de cirurgiões

\footnotetext{
${ }^{1}$ Médico Veterinário, Professor Assistente, Departamento de Clínica Médica Veterinária, Universidade Federal de Mato Grosso, Doutorando em Cirurgia Experimental, Universidade Federal de Santa Maria (UFSM), Camobi, 97105-900, Santa Maria - RS. E-mail: eacont@terra.com.br. Autor para correspondência.

${ }^{2}$ Médico Veterinário, Mestre, Professor Assistente, Departamento de Patologia, Universidade Federal de Santa Maria (UFSM).

${ }^{3}$ Médico Veterinário, Mestre, Professor Assistente, Faculdade de Veterinária, Universidade Federal do Rio Grande do Sul.

${ }^{4}$ Médico Veterinário, PhD., Professor Adjunto, Departamento de Clínica de Pequenos Animais (DCPA), UFSM.

${ }^{5}$ Médico Veterinário, Doutor, Professor Titular, DCPA, UFSM.

Recebido para publicação em 29.08.00. Aprovado em 06.12.00
} 
veterinários, principalmente no que tange à qualidade cicatricial promovida após a traqueoplastia. Para tanto, várias técnicas e materiais têm sido desenvolvidos como arcabouço ou para serem incorporadas à parede traqueal, no intuito de diminuir os efeitos cicatriciais indesejáveis. A proliferação de tecido conjuntivo de granulação no sítio da anastomose (SMITH et al., 1990; NAKANISHI $\boldsymbol{e t} \boldsymbol{a l} ., 1998$; TOJO $\boldsymbol{e t} \boldsymbol{a l} ., 1998)$ e a constrição/estenose (SABÁS et al., 1977; PETROIANU \& BARBOSA, 1993; MUKAIDA et $a l .$, 1998) foram considerados como sendo grandes empecilhos à cicatrização.

Para ECKERSBERGER et al. (1987), os pontos básicos para a aplicação clínica com longa durabilidade, de um substitutivo de segmento traqueal, exige estabilidade mecânica, completa capacidade de repitelização e ausência de tecido de granulação. Para COSTANTINO et al. (1992), há ainda a necessidade de esse substitutivo ser vascularizado ou permitir a neovascularização, ser estruturalmente rígido para manter o lume traqueal durante a ventilação e ter biocompatibilidade em caso de materiais sintéticos.

Alguns pesquisadores desenvolveram trabalhos no intuito de testar ou atender tais exigências. A cartilagem autógena de pavilhão auricular foi utilizada em coelhos por CHEHUEN NETO $\boldsymbol{e t}$ al. (1991). Em cães, foram utilizados a cartilagem costal associada a tubo de silicone por ECKERSBERGER et al. (1987); a traquéia homógena conservada em glutaraldeido por PINTO et al. (1992) e PETROIANU \& BARBOSA (1993); o enxerto autógeno revascularizado de jejuno associado a tubo de dacron por COSTANTINO et al. (1992); a artéria aorta abdominal conservada em glutaraldeido por PETROIANU \& BARBOSA (1993); o segmento livre de peritônio muscular por EURIDES \& RONCHI (1994); placa de silicone por CONTESINI et al. (1995); traquéia homógena conservada em glicerina por DALECK et al. (1998) e aloenxerto crioconservado por MUKAIDA et al. (1998). Esses trabalhos apresentaram resultados heterogêneos, com diferentes índices de qualidade do material substitutivo.

Diante disso, objetivou-se observar o comportamento cicatricial da pina como enxerto autógeno ou como implante homógeno conservado em glicerina a $98 \%$, quando aplicada na correção de defeito induzido experimentalmente na parede traqueal cervical em cães.

\section{MATERIAL E MÉTODOS}

Para a experimentação, foram utilizados doze cães, adultos, hígidos, cinco machos e sete fêmeas, com peso entre 11 a $15 \mathrm{~kg}$. O protocolo anestésico foi constituído pela administração de acepromazina, na dose de $0,1 \mathrm{mg} / \mathrm{kg}$ de massa corporal, intra-venosa, na pré-anestesia, seguido da administração de tiopental sódico na dose de $12,5 \mathrm{mg} / \mathrm{kg}$, intravenoso para indução anestésica e manutenção com halotano em sistema circular através de calibrador universal.

Os animais foram separados em dois grupos experimentais: GI constituído por 6 animais, os quais foram submetidos ao enxerto autógeno de cartilagem da pina e GII, também constituído por 6 animais, submetidos à implante homógeno do mesmo tipo de estrutura conservada em solução de glicerina a $98 \%$.

Imediatamente antes da cirurgia, os cães foram higienizados e submetidos a tricotomia na região cérvico-ventral. Os do grupo I também foram depilados na região auricular. A conchectomia parcial bilateral foi realizada nos cães do grupo I, sendo que parte do material foi destinado ao transplante, permanecendo submersa em solução isotônica de cloreto de sódio $(\mathrm{NaCl})$ morna, até o momento da sua aplicação, enquanto o restante foi destinada à conservação em solução de glicerina a $98 \%$ à temperatura ambiente, por um período mínimo de 30 dias. No momento da execução da cirurgia do grupo II, o segmento de cartilagem foi retirado da glicerina a $98 \%$, lavado com solução isotônica de $\mathrm{NaCl}$, morna e mantida sob imersão nesse mesmo tipo de solução, porém acrescida de ampicilina sódica $500 \mathrm{mg}$, por aproximadamente trinta minutos até a sua implantação.

Os animais foram acomodados em decúbito dorsal, com a região do pescoço apoiada num coxim para mantê-lo levemente distendido, facilitando o acesso cirúrgico que foi, em ambos os grupos, cérvico-medial. Para tal, os grupos musculares esternohioideo e esternotiroideo foram afastados, externando um segmento traqueal sem, no entanto, separá-lo completamente do seu leito anatômico. A seguir foi removido um segmento da traquéia em dimensões de três anéis traqueais em seu comprimento e um terço na sua circunferência na região ventral.

A traqueoplastia foi efetuada com a fixação de um retalho confeccionado da cartilagem da pina autógena a fresco, no grupo I e da cartilagem da pina homógena conservada em glicerina a $98 \%$, no grupo II. A dimensão do retalho foi compatível com a do defeito provocado. O padrão de sutura utilizado para esse procedimento foi o simples interrompido incluindo a mucosa traqueal, com fio de náilon 4-0 monofilamentar agulhado (Figura 1).

Ao término da síntese da cartilagem, o campo operatório foi lavado de forma abundante, 


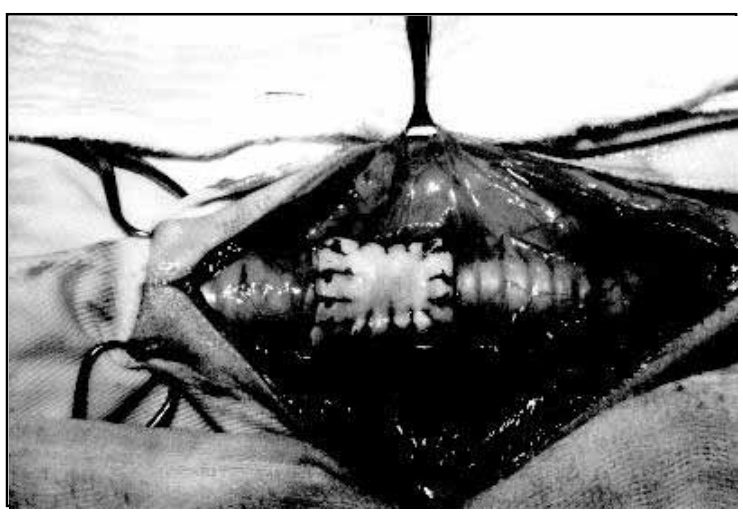

Figura 1 - Traqueoplastia em cães. Retalho de cartilagem de pina autógena, em tamanho compatível com o defeito traqueal.

com solução de $\mathrm{NaCl}$ 0,9\% morna. A seguir foram reaproximados os grupos musculares, reduzido o espaço morto e executada a dermorrafia em padrão simples interrompido com fio poliamida 0-20. Durante o período pós operatório imediato, os animais receberam flunixim meglumine na dose de $1,0 \mathrm{mg} / \mathrm{kg}$, intra-muscular, a cada 24 horas, durante três dias consecutivos e curativo local com solução de polivinilpirrolidona (PVP-I) $1 \%$ em solução isotônica de $\mathrm{NaCl}$.

Os cães foram avaliados clinicamente em estado de repouso e sob exercício físico com caminhadas moderadas diárias, por quinze minutos, durante 30 e 45 dias de acordo com cada subgrupo. Ao final desses períodos, foram coletados os segmentos traqueais com a área de transplante ou implante, para avaliação macroscópica e em microscopia óptica. Os fragmentos foram processados, cortados e corados em hematoxilinaeosina.

\section{RESULTADOS E DISCUSSÃO}

A substituição parcial da parede traqueal tem sido alvo de vários experimentos dentro da medicina veterinária e humana. Algumas tentativas foram realizadas com o uso de materiais prostéticos sintéticos, diferentes tecidos autógenos e homógenos, tanto em cirurgias experimentais como em aplicações clínicas. Dessa forma a cartilagem da pina é apresentada como uma alternativa experimental de possível aplicabilidade prática na correção de defeitos da parede traqueal cervical em cães.

O protocolo anestésico e o acesso cirúrgico ventro-medial utilizados, seguindo sugestão de CONTESINI et al. (1995), demonstraram-se eficazes para o procedimento operatório, uma vez que o tempo médio utilizado para a execução do transplante/implante foi de 30 minutos, descontando-se o tempo de coleta de material para o grupo I e de reidratação da cartilagem para o grupo II. O acesso cirúrgico permitiu, ainda, que os dois terços remanescentes da circunferência traqueal fosse mantido em conexão com o tecido adjacente bastante vascularizado, mantendo significativo o fluxo sangüíneo local. Esse cuidado, que deve ter favorecido o processo cicatricial, corrobora com a preocupação básica dos princípios da cicatrização relatado por COSTANTINO et al. (1992) que consideraram imprescindível a vascularização local para o processo cicatricial. KAWAHARA et al. (1998), usando a omentopexia no sítio de anastomose traqueal, e NAKANISHI et al. (1998), utilizando o músculo Latissinius dorsi, confirmaram a importância de favorecer o afluxo sangüíneo no local da anastomose traqueal.

Durante o período de observação e avaliação, os animais não apresentaram quaisquer sinais de incômodo pela presença da cartilagem transplantada/implantada, ou mesmo dos fios de sutura, mesmo em circunstâncias de exercício físico moderado. Esses sinais poderiam ser apresentados em forma de tosse, de acordo com o relato feito por DALECK et al. (1998), nos primeiros dias após a cirurgia. A ausência desses sinais provavelmente tenha sido em decorrência do caráter não circunferencial, neste experimento.

A performance respiratória manteve-se em qualidade satisfatória durante todo o período de observação, não sugerindo quaisquer alterações significativas na patência do lume traqueal, como estenose cicatricial por contração ou granulação exuberante. Esse fato foi confirmado na avaliação macroscópica da área operada ao final dos período de observação. A inexistência de estenose clínica corrobora com os fundamentos de caracterização da qualidade do material empregado na correção da parede traqueal, para que haja qualidade satisfatória na vida do animal, conforme afirmaram SABÁS et al. (1977), PETROIANU \& BARBOSA (1993), GRIFFITHS et al. (1998). Tais informações também foram encontradas nos relatos de exuberante granulação na anastomose, encontrada por PINTO et al. (1992) em traquéias homólogas conservadas em glutaraldeído, NAKANISHI et al. (1998) com enxerto traqueal autógeno e TOJO $\boldsymbol{e t}$ al. (1998) com aloenxerto de traquéias a fresco. A manutenção da patência respiratória do animal foi considerada por esses pesquisadores, como o grande empecilho na qualidade de seus resultados.

A superfície do lume traqueal na área da cirurgia apresentou-se, macroscopicamente, com 
aspecto uniforme em relação às demais, com uma estrutura esbranquiçada e delgada encobrindo a cartilagem da pina, sugerindo migração do epitélio por toda a superfície do retalho (Figura 2a). Esse fato foi confirmado pela microscopia, revelando completa repitelização local (Figura 2b), com focos de hiperplasia epitelial nos animais do grupo I, compatível com os resultados observados por NAKANISHI $\boldsymbol{e t}$ al. (1998). Isso exprime, mais uma vez, a importância da vascularização local e/ou a permissividade de neovascularização, conforme relataram COSTANTINO et al. (1987), uma vez que CONTESINI et al. (1995) não presenciaram completa migração epitelial, num experimento utilizando placa de silicone. Também, o tempo necessário para que a proliferação do epitélio se concretize parece ter sido adequado, uma vez que a partir de 30 dias de observação, o local já estava regenerado. Embora o trabalho de CHEHUEN NETO et al. (1991), que utilizaram cartilagem de pavilhão auricular transplantado para a traquéia em coelhos, não tenha demonstrado completa regeneração epitelial ao final de 21 dias.

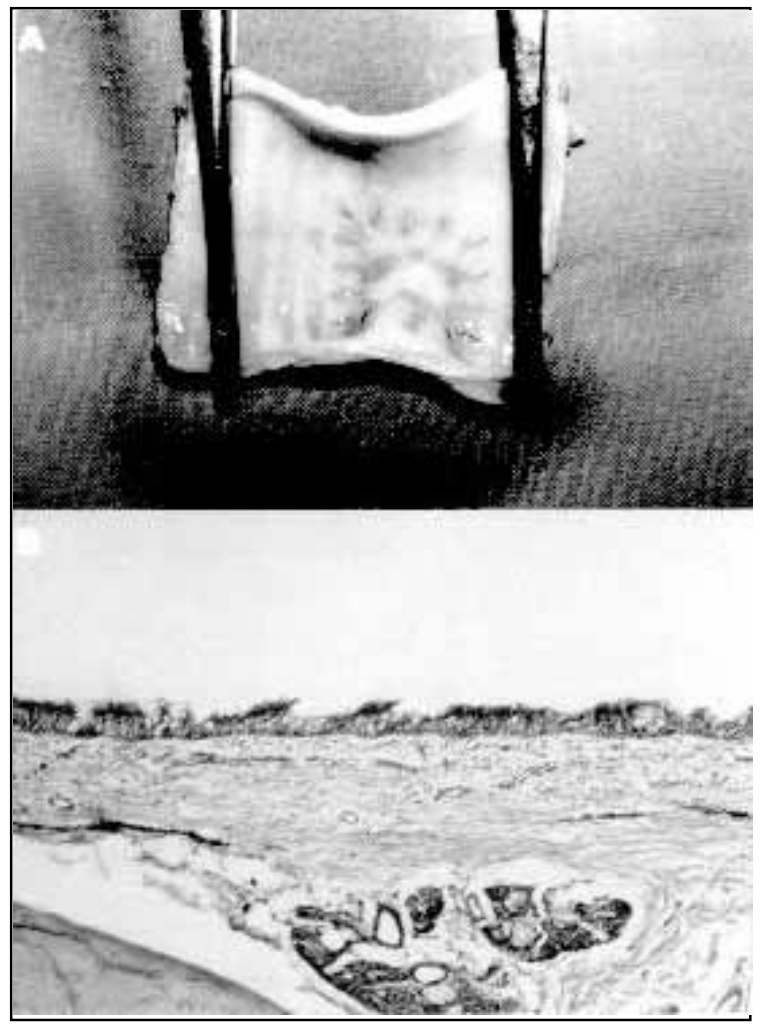

Figura 2 - Traqueoplastia em cães. Avaliação do lume traqueal no sítio da fixação do retalho de cartilagem da pina. a) na macroscopia, a superfície interna do retalho é encoberta por uma estrutura delgada esbranquiçada; b) na microscopia, revestimento interno do retalho pelo epitélio ciliado, característico da traquéia.
Os pontos de sutura, na avaliação macroscópica, não eram evidentes na face intraluminal da área operada, nos períodos finais de observação. Isso sugere que há uma alta capacidade regenerativa do epitélio traqueal, encobrindo-o, embora não se tenha assumido o compromisso em não atingir o lume traqueal, como relataram EURIDES \& RONCHI (1994), na restauração traqueal com segmento livre de peritônio-muscular em cães.

$\mathrm{Na}$ avaliação microscópica, foram observadas, ainda, moderada infiltração de neutrófilos e alguma fibrose na lâmina própria dos animais do grupo I, discreta invasão de macrófagos e vasos neoformados, sugerida como fator de regeneração por NAKANISHI et al. (1998) e discreta reação inflamatória na lâmina própria nos animais do grupo II.

\section{CONCLUSÃO}

Os resultados obtidos permitem indicar o uso de cartilagem da pina como enxerto autógeno e também de implante homógeno conservado em glicerina a $98 \%$, em forma de retalho, para corrigir defeitos da parede traqueal comprometendo parcialmente até 3 anéis traqueais, embora, histologicamente, a cartilagem conservada em glicerina a 98\%, tenha induzido melhor reação cicatricial.

\section{REFERÊNCIAS BIBLIOGRÁFICAS}

CHEHUEN NETO, J.A., NIGRO, A.J.T., BELMONTE NETTO, L., et al. Restauração traqueal com enxerto cartilaginoso autólogo de pavilhão auricular: estudo experimental em coelhos. Acta Cirúrgica Brasileira, v.6, n.4, p.169-176, 1991.

CONTESINI, E.A., PIPPI, N.L., SANTOS, S.C., et al. Implante de placa de silicone na substituição parcial da parede traqueal em caninos. Ciência Rural, v.25, n.3, p.417-420, 1995.

COSTANTINO, M.P.D., NUSS, D.W., SNYDERMAN, C.H., $\boldsymbol{e}$ al. Experimental tracheal replacement using a revascularized jejunal autograft with an implantable dacron mesh tube Annals of Otology, Rhinology \& Laringology, v.101, p.807-814, 1992 .

DALECK, C.R., COSTA NETO, J.M., ALESSI, A.C. Prótese traqueal em cães com traquéia homóloga. estudo experimental. Veterinária Notícias, v.4, n.1, p.57-62, 1998.

ECKERSBERGER, F., MORITZ, E., WOLNER, E. Circunferential tracheal replacement with costal cartilage. Journal of Thoracic and Cardiovascular Surgery, v.94, n.2, p.175-180, 1987.

EURIDES, D., RONCHI, C.P.H. Restauração da traquéia de cães com segmento livre de peritônio-muscular. estudo experimental. Acta Cirúrgica Brasileira, v.9, n.4, p.195200, 1994. 
GRIFFITHS, L.G., SULLIVAN, M., LERCHE, P. Intrathoracic tracheal avulsion and pseudodiverticulum following pneumomediastinum in a cat. Veterinary Record, v.142, p.693-696, 1998.

KAWAHARA, K., INUTSUKA, K., HIRATSUKA, M., et $\boldsymbol{a l}$. Tracheal transplantation for carinal reconstruction in dogs. Journal of Thoracic and Cardiovascular Surgery, v.116, n.3, p.397-401, 1998 .

MUKAIDA, T., SHIMIZU, N., AOE, M., et al. Experimental study of tracheal allotransplantation with cryopreserved grafts. Journal of Thoracic and Cardiovascular Surgery, v.116, n.2, p.262-266, 1998.

NAKANISHI, R., HASHIMOTO, M., YASUMOTO, K. Improved airway healing using basic fibroblast growth factor in a canine tracheal autotransplantation model. Annals of Surgery, v.227, n.3, p.446-454, 1998.

PETROIANU, A., BARBOSA, A.J.A. Experimental reconstruction of anterior and circumferential defects of the cervical trachea. The Laryngoscope, v.103, n.11, p.12591263, 1993.

PINTO, M.A., CARDOSO, G., MARCHEVISK, R.S., et al. Bioprótese traqueal. Ciência Rural, v.22, n.3, p.293-298, 1992.

SABÁS, A.A., UEZ, J.B., ROJAS, O., et al. Replacement of the trachea with dura mater - experimental work. Journal of Thoracic and Cardiovascular Surgery, v.74, n.5, p.761$765,1977$.

SMITH, M.M., GOURLEY, I.M., AMIS, T.C., et al. Management of tracheal stenosis in dogs. Journal of the American Veterinary Medical Association, v.196, p.931934, 1990.

TOJO, T., NIWAYA, K., SAWABATA, N., et $\boldsymbol{a l}$. T racheal replacement with cryopreserved tracheal allograft: experient in dogs. Annals of Thoracic Surgery, v.66, n.1, p.209-213, 1998.

Ciência Rural, v. 31, n. 4, 2001. 